\title{
Technische Aspekte der Beatmungsmedizin
}

\section{Technical Aspects of Mechanical Ventilation}

D. Heyse, G. Schürholz, S. Böckling, K. Siemon, D. Dellweg

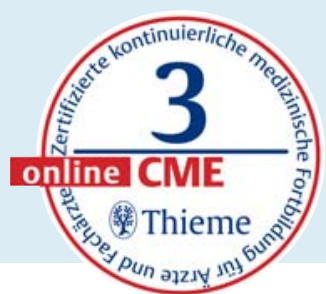

Klinik für Pneumologie, Krankenhaus Kloster Grafschaft, Schmallenberg

\section{Lernziele}

$\nabla$

Nach DRG-Statistik wurden in Deutschland im Jahr 2010369730 Patienten über insgesamt 61.035.542 Stunden beatmet [1]. Die Vielfalt der dafür eingesetzten Beatmungsgeräte ist groß. Dieser Artikel versucht wesentliche technische Aspekte der Funktionsweise von Beatmungsgeräten herauszuarbeiten, um dem Nutzer ein tieferes Verständnis in der Anwendung zu ermöglichen. Lernziel sollte sein, Kenntnisse über die Druckund Flusssteuerung invasiver Beatmungsgeräte zu erlangen. Der Artikel zeigt auf, wie die Beatmungsluft zum Patienten kommt und wie die Einstellung am Beatmungsgerät auf technischer Ebene umgesetzt wird.

\section{Einleitung}

Beatmungsgeräte werden heutzutage breit eingesetzt und wie selbstverständlich genutzt. Beatmungsgeräte unterliegen dem nationalen Medizinproduktegesetz, der Medical Device Directive (MDD-Richtlinie 93/42) der EU, sowie den DINNormen der EU (DIN EN 60601-2-12 und DIN EN 60601-1-8). Sie werden von den meisten Benutzern als sicher erachtet. Trotzdem findet man in der Literatur vielfache Hinweise für fatale Ereignisse bei beatmeten Patienten, hervorgerufen durch technisches und/oder menschliches Versagen $[2-14]$.

Die Hauptursache für fatale Ereignisse während einer Beatmung sind dabei unzureichende Kenntnisse über das verwendete Equipment und dessen Funktion [15]

Die Güte einer Beatmung richtet sich nach einer Reihe von Kriterien, deren Prioritäten vom Arzt festzulegen sind. Hierzu gehören die Zielparameter des Gasaustausches (im Wesentlichen $\mathrm{PaO}_{2}, \mathrm{PaCO}_{2}$ und der $\mathrm{pH}$ Wert), das Vermeiden von Schäden an Lunge und Atemmuskulatur, die Entlastung der Atemmuskulatur sowie der Patientenkomfort.
Aus der Komplexität der an die Beatmung gestellten Ansprüche wird schnell ersichtlich, dass eine explizite Kenntnis der Funktionsweise von Ventilatoren für die Umsetzung der Ziele erforderlich ist. Die oben genannten Autoren haben in Vorbereitung auf diesen Artikel zwei Beatmungsgeräte verschiedener Hersteller und Bauart mit den Technikern der jeweiligen Firmen zerlegt und die Funktionsweise mit Hilfe von Schaltplänen nachvollzogen. Hierher stammt auch das verwendete Bildmaterial. Die hier abgedruckten technischen Beschreibungen allgemeiner Funktionsprinzipien sind daher exemplarisch und zeigen wahrscheinlich nicht den vollen Umfang aller technischen Umsetzungsmöglichkeiten.

\section{Die Bedienungseinheit (User Interface Module) \\ $\nabla$}

Dieses System besteht in der Regel aus einer Kombination von Display und Bedienungselementen und ermöglicht die Interaktion von Bediener und Beatmungsgerät. Die hier vorgewählten Einstellungen werden dann über Mikroprozessoren und eine entsprechende Software im pneumatischen System des Beatmungsgerätes umgesetzt. Die Bedienungseinheit sollte einfach und intuitiv zu bedienen sein und einen logischen Aufbau haben. Eine versehentliche Verstellung der Parameter sollte ausgeschlossen werden.

Die Bedienungseinheit sollte intuitiv zu bedienen sein, eine akzidentelle Verstellung der Parameter sollte ausgeschlossen sein.

\section{Das luftführende System \\ $\nabla$}

Das luftführende oder pneumatische System regelt den Fluss und den Druck des Atemgases.

Das System besteht aus einer externen und/oder internen Einheit, die den Druck generiert, und einer Einheit, die die inspiratorische Sauerstoff-
VNR

2760512014144213377

Bibliografie

Dol http://dx.doi.org/

10.1055/s-0034-1378072

Pneumologie 2014; 68: 811-820

(c) Georg Thieme Verlag KG

Stuttgart · New York

ISSN 0934-8387

Korrespondenzadresse PD Dr. med. Dominic Dellweg Pneumologie, Allergologie, Beatmungs- und Schlafmedizin Fachkrankenhaus Kloster Grafschaft Annostr. 1 57392 Schmallenberg d.dellweg@fkkg.de 

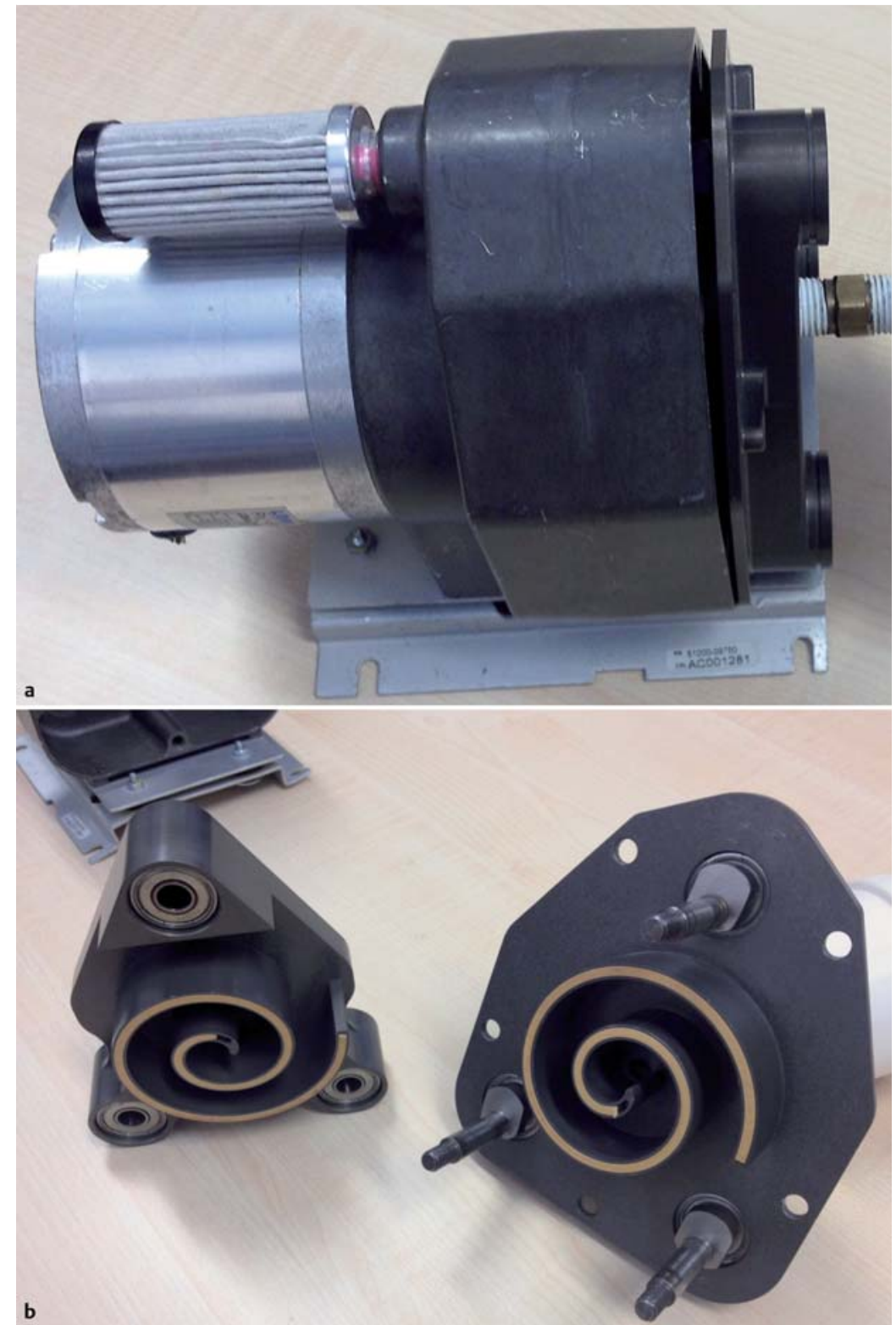

Abb. 1 a Kompressor-Einheit eines Beatmungsgerätes (Avea, CareFusion). Teil b der Abbildung zeigt die zerlegte Kompressoreinheit. Die beiden schneckenartigen Bauteile rotieren ineinander und verdichten so die zugeführte Raumluft.

konzentration regelt (Blender/Mischer). Eine Steuerung regelt Druck und Fluss gemäß der vorselektierten Beatmungseinstellung.

Durch das luftleitende System gelangt das Atemgas von der Druckquelle zum Patienten. Druck, Fluss und Sauerstoffkonzentration werden hier geregelt.

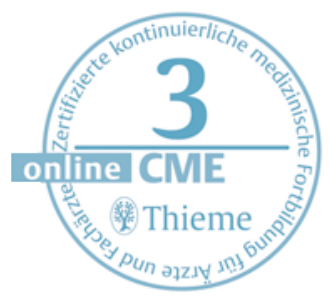

\section{Gasdruckentstehung}

$\nabla$

Die für die Beatmung notwendige Druckluft wird entweder im Gerät durch einen Kompressor erzeugt oder stammt aus dem stationären Druckluftsystem des Krankenhauses bzw. der versorgenden Einrichtung. Medizinische Atemgase un-
Tab. 1 Zusammensetzung medizinischer Gase nach dem Europäischen Arzneibuch.

\begin{tabular}{|l|l|}
\hline Bestandteil & Zulässiger Wert \\
\hline $\mathrm{CO}_{2}$ & bis $500 \mathrm{ppm}$ \\
\hline $\mathrm{CO}$ & bis $5 \mathrm{ppm}$ \\
\hline $\mathrm{SO}_{2}$ & bis $1 \mathrm{ppm}$ \\
\hline $\mathrm{O} \mathrm{L}$ & bis $0,1 \mathrm{mg} / \mathrm{m}^{3}$ \\
\hline $\mathrm{NO} / \mathrm{NO}_{2}$ & bis $2 \mathrm{ppm}$ \\
\hline $\mathrm{Wasser}$ & bis $67 \mathrm{ppm}$ \\
\hline $\mathrm{O}_{2}$ & 20,4-21,4\% \\
\hline $\mathrm{CO}_{2}$ & bis $300 \mathrm{ppm}$ \\
\hline $\mathrm{CO}$ & bis $5 \mathrm{ppm}$ \\
\hline Wasser & bis $67 \mathrm{ppm}$ \\
\hline $\mathrm{O}_{2}$ & min 99,5 \\
\hline
\end{tabular}

terliegen seit dem 1.8.2001 dem Arzneimittelgesetz. Die Anforderungen an die Zusammensetzung medizinischer Gase, die auch für den Betrieb von Beatmungsgeräten eingesetzt werden, sind im Europäischen Arzneibuch veröffentlicht ( $\odot$ Tab. 1, [16]). Erstaunlich ist dabei, dass es keine Vorschriften für die Temperatur der abgegebenen Gase gibt. Ortsfeste Gasversorgungsanlagen medizinischer Einrichtungen arbeiten in der Regel mit einem Betriebsdruck von 5 bar und werden vom Wandanschluss über spezielle Druckschläuche mit verwechslungssicheren Konnektoren für Druckluft und Sauerstoff an das Beatmungsgerät angeschlossen.

Neben Beatmungsgeräten, die ausschließlich mit extern zugeführter Druckluft arbeiten, gibt es Geräte, die mit einem eingebauten Kompressor selber Druckluft erzeugen können und somit unabhängig vom Druckluftsystem der medizinischen Einrichtung arbeiten können. Da diese Kompressoren lediglich Raumluft verdichten, können ohne externe Sauerstoffzufuhr aus dem Wandanschluss keine Sauerstoffkonzentrationen oberhalb Raumluftniveau (21\%) erreicht werden. - Abb. 1 zeigt den Aufbau und die Wirkungsweise eines solchen integrierten Kompressors.

Als Druckquelle dient je nach Gerät der Druckluft/ Sauerstoff-Wandanschluss oder/und ein interner Kompressor. Für Sauerstoffkonzentrationen oberhalb von $21 \%$ ist ein externer Sauerstoffanschluss erforderlich.

\section{Arbeitsdruck im Beatmungsgerät \\ $\nabla$}

Der aus dem Wandanschluss kommende Druck für Luft und Sauerstoff wird am Eingang des Beatmungsgerätes zunächst gemessen und dann durch einen Druckminderer auf den im Gerät verwendeten Arbeitsdruck reduziert. Aus Sicherheitsgründen und um den Verschleiß zu minimieren, sollte der Arbeitsdruck so gering wie möglich sein. Andererseits muss der Arbeitsdruck ausreichend hoch sein, um den nötigen Inspirationsfluss zum Patienten bedienen zu können. Um 
dies gewährleisten zu können, scheint ein Arbeitsdruck von 1,72 bar (25 PSI) ausreichend zu sein [17]. Werden niedrigere Drücke verwendet, so kann über einen patientennahen zusätzlichen Druckspeicher (Akkumulator) ein ausreichender Fluss des Atemgases zum Patienten gewährleistet werden. So kann dann z. B. ein Arbeitsdruck von 0,76 bar (11 PSI) ausreichend sein. $\bullet$ Abb. 2 zeigt exemplarisch einen solchen Druckspeicher, vom Prinzip her handelt sich dabei um ein zusätzliches Reservoir, das einen plötzlichen Druckabfall im System (z. B. bei schneller Inspiration durch den Patienten) verhindert. Damit in jedem Fall die am Beatmungsgerät eingestellte inspiratorische Sauerstoffkonzentration auch zum Patienten abgegeben wird, muss der Druckspeicher immer mit der aktuell eingestellten Sauerstoffkonzentration durchflutet werden [18].

Der geräteintern verwendete Druck muss ausreichend hoch sein, um dem Bedarf des Patienten zu genügen. Ein interner Druckspeicher kann hier zusätzliche Reserven liefern.

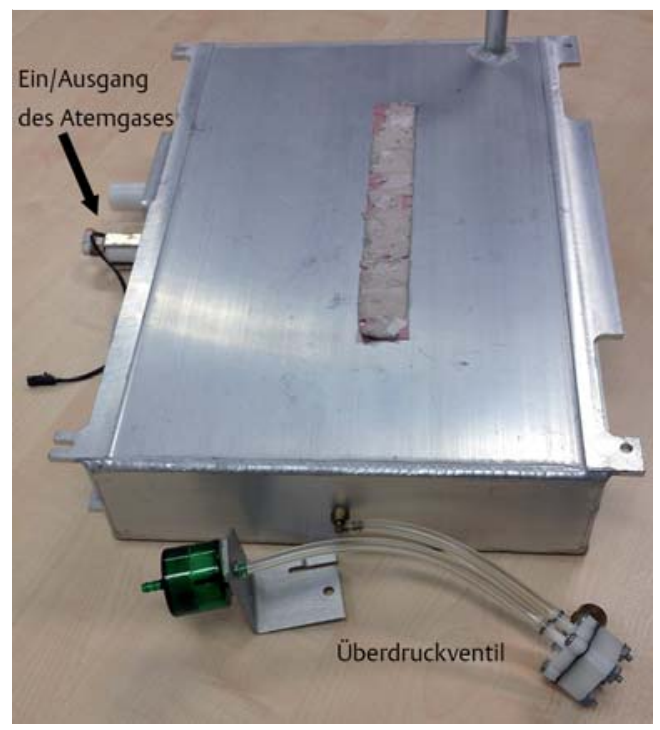

Abb. 2 Druckspeicher (Akkumulator) mit einem Volumen von 4,6 Litern (Avea, CareFusion).

\section{Luftleitung im Beatmungsgerät \\ $\nabla$}

Um die Luft von der Druckquelle zu den verschiedenen Bauteilen des Beatmungsgerätes und schließlich zum Patienten zu leiten, braucht man ein luftleitendes System.

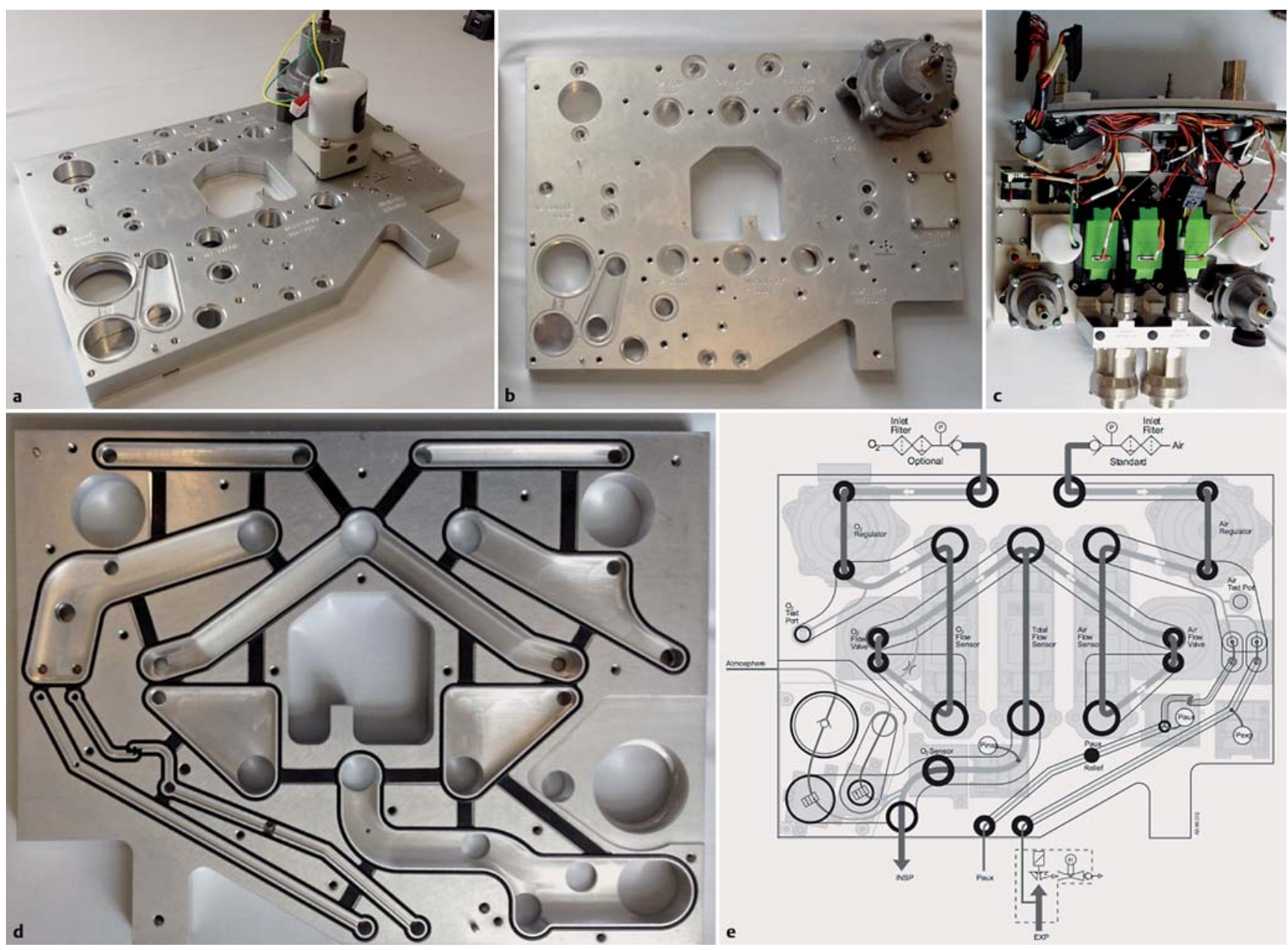

Abb. 3 Aluminiumbodenplatte (Oberseite (a und b), mit aufgeschraubten Komponenten (c), von der Unterseite mit eingefrästen Luftkanälen (d) sowie als schematische Darstellung des luftleitenden Systems (e), Engström Carestation, GE). 


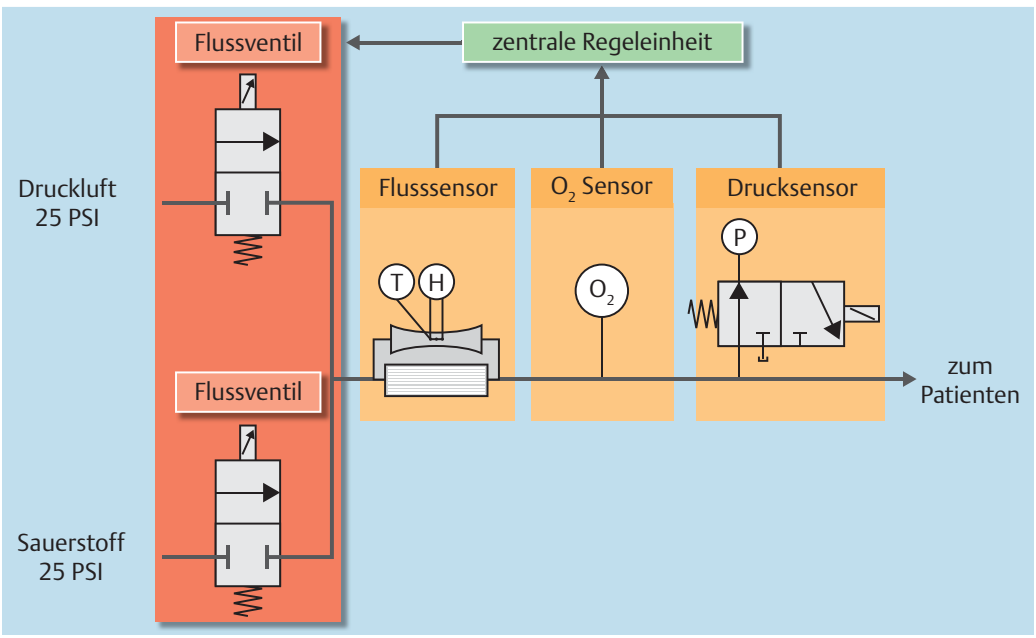

Abb.4 Die beiden Flussventile regeln nicht nur das richtige Verhältnis von Druckluft und Sauerstoff, sondern regulieren auch schon den Druck und den Fluss, der zum Patienten weitergeleitet wird. Hierfür ist eine hochfrequente Kommunikation zwischen den nachgeschalteten Sensoren, der zentralen Regeleinheit und den Flussventilen erforderlich (vereinfachte ausschnittsweise Darstellung des Schaltplanes der Engström Carestation, GE). Der rot hinterlegte Bereich stellt den Bereich dar, der als „Blender“ (Mischer) bezeichnet wird.

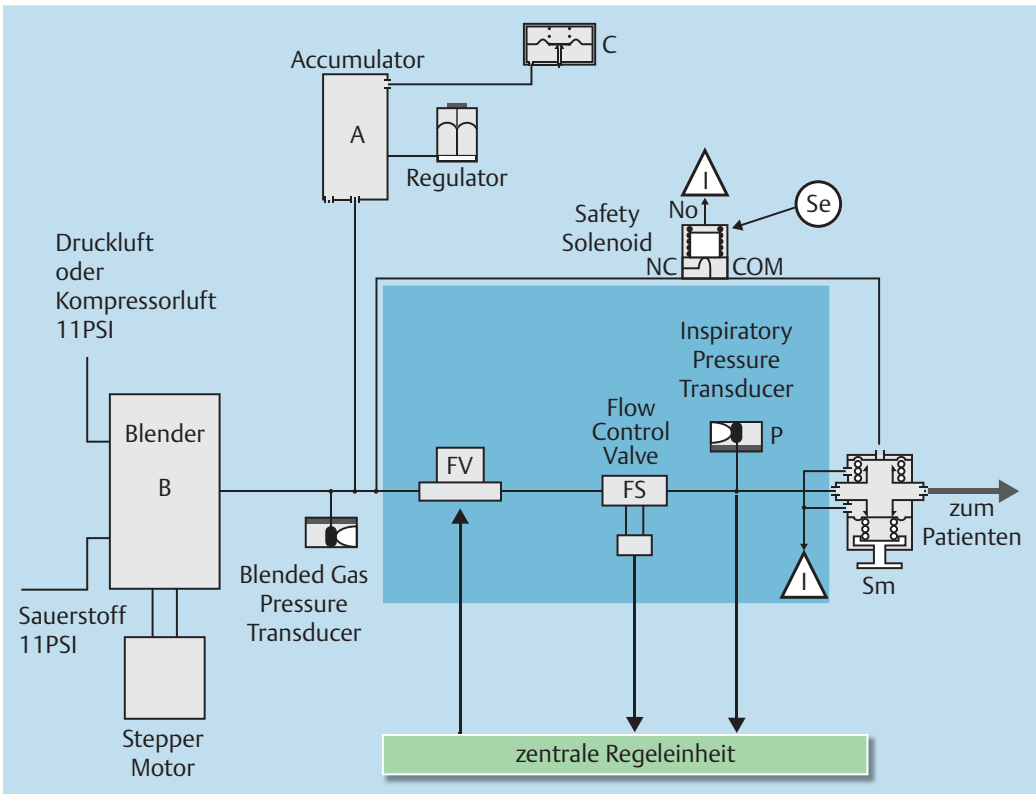

Abb.5 Im Mischer (Blender, B) wird die inspiratorische Sauerstoffkonzentration eingestellt. Der Druckspeicher (Akkumulator, A) dient als Druckreserve und wird ständig mit dem Gemisch aus dem Blender durchflutet. Um dies zu gewährleisten, wird ein Auslass-Ventil (C) benötigt. Danach wird im blau hinterlegten Bereich der Fluss und der Druck der zum Patienten gehenden Atemluft geregelt. Hierzu sind ein Flussventil (FV), ein Flusssensor (FS) und ein Drucksensor (P) erforderlich. Zusätzlich eingezeichnet sind ein elektronisches (Se) und ein mechanisches (Sm) Sicherheitsventil (vereinfachte ausschnittsweise Darstellung des Schaltplanes der Avea, CareFusion).

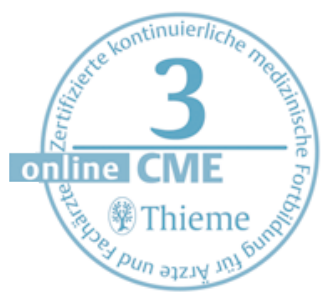

Dies kann klassisch über Schläuche zwischen den einzelnen Komponenten oder über eine Luftkanal-führende Bodenplatte im Beatmungsgerät realisiert werden.

- Abb. 3 zeigt die eine aus Aluminium gefräste Bodenplatte, in der die gesamte Luftleitung innerhalb des Gerätes stattfindet. Die entsprechenden Bauteile sind auf dieser Platte verschraubt und regeln die Leitung des Atemgases. Der Strömungs- widerstand des luftleitenden Systems muss so gering sein, dass der im Gerät verwendete Druck einen ausreichenden Gasfluss zum Patienten gewährleistet.

Die geräteinterne Luftleitung kann über Schlauchsysteme oder feste Kanäle erfolgen.

Ihr Strömungswiderstand sollte gering sein.

\section{Regulation der inspiratorischen Sauerstoffkonzentration}

$\nabla$

Die inspiratorische Sauerstoffkonzentration wird im Blender (Mischer) des Beatmungsgerätes auf die vorgewählte Konzentration gemischt. Hierfür werden die Sauerstoff- und Druckluftzufuhr entsprechend durch ein Flussventil im richtigen Verhältnis geregelt bzw. gemischt. Beatmungsgeräte, die ohne internen Druckspeicher arbeiten, können durch die Flussventile an dieser Stelle bereits den Druck und den Fluss steuern, der zum Patienten weitergegeben wird. Hierzu kommunizieren diese Flussventile mit einem nachgeschalteten Drucksensor und einem nachgeschalteten Flusssensor, die direkt vor dem zum Patienten führenden Inspirationsschenkel liegen ( $\bullet$ Abb.4).

Hat das Beatmungsgerät einen internen Druckspeicher, so muss dieser hinter den Regelventilen liegen, die die inspiratorische Sauerstoffkonzentration mischen, da der Druckspeicher ja immer mit der aktuellen Sauerstoffkonzentration versorgt werden muss. Eine Steuerungseinheit, bestehend aus Flussventil, Flusssensor und Drucksensor ( $\bullet$ Abb. 5, blau hinterlegt), liegt dann, in Flussrichtung gesehen, hinter dem Anschluss für den internen Druckspeicher und steuert den Fluss bzw. Druck zum Patienten ( Abb.5).

Im Blender wird Sauerstoff und Druckluft über Flussventile im eingestellten Verhältnis gemischt. Wird ein interner Druckspeicher verwendet, so muss dieser immer mit der aktuellen $\mathrm{FiO}_{2}$ befüllt sein und somit hinter dem Blender liegen.

\section{Steuerung des Atemgases zum Patienten} $\nabla$

Eine Steuerungseinheit für das zum Patienten geleitete Atemgas muss sowohl den Druck als auch den Fluss messen und regulieren können. Die Steuerung des Atemgasstromes erfolgt über ein oder mehrere Flussventile in Kommunikation mit nachgeschalteten Druck- und Flusssensoren (blauer Bereich der $\bullet$ Abb.5). Die Kommunikation erfolgt über die zentrale Recheneinheit in Zeitintervallen von wenigen Millisekunden. Den Aufbau eines geregelten Flussventils zeigt $\bullet$ Abb.6. In - Abb.7 ist die Möglichkeit verschiedener Verfahren zur Flussmessung dargestellt. Wird ein Flusssensor verwendet, der nach dem Prinzip 


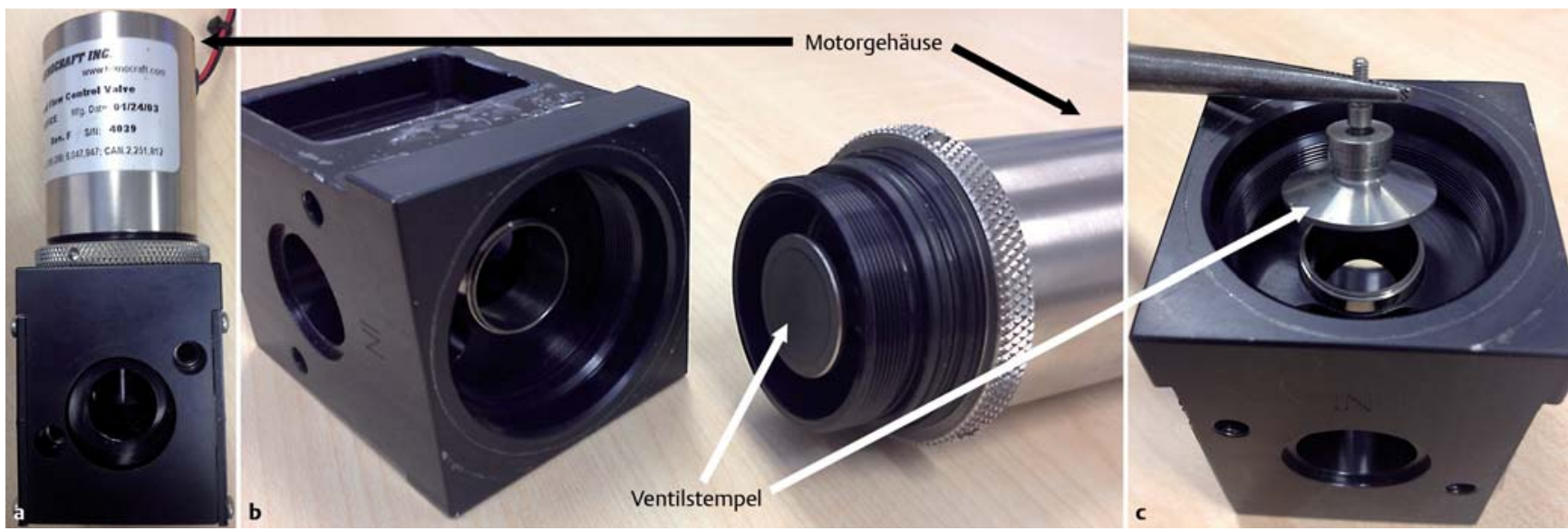

Abb. 6 Zeigt exemplarisch den Aufbau eines Flussventils. Ein Motor mit linearer Regelung regelt den Luftstrom über einen Ventilstempel. a Komplettes Ventil. b Hier wurde der Motor mit Gehäuse von der luftleitenden Einheit getrennt. c zeigt den vom Motor getrennten Stempel auf der luftleitenden Einheit. (Die abgebildeten Komponenten stammen aus dem Beatmungsgerät Avea, CareFusion).

eines Pneumotachografen arbeitet $(\bullet$ Abb.7a), können Zusatzinformationen wie Gasdichte und Gastemperatur, die manuell eingegeben bzw. geräteintern gemessen werden, die Messgenauigkeit des Flusssensors verbessern.

Eine Regeleinheit besteht aus Flussventil, Flusssensor und Drucksensor.

\section{Druck- und volumenkontrollierte Beatmung}

$\nabla$

Bei der volumenkontrollierten Beatmung (VCV = Volume Controlled Ventilation) wird ein voreingestelltes Volumen mit jedem maschinellen Atemzug abgegeben. Dabei wird über eine festgelegte Zeitdauer (Inspirationszeit=Ti) ein konstanter Fluss verwendet.

Alternativ zu einem konstanten Fluss können je nach Hersteller auch abfallende Flussprofile gewählt werden. Die Höhe des Atemwegdruckes, der für die Verabreichung des Tidalvolumens erforderlich wird, variiert und hängt von der Compliance und Resistance von Thorax und Lunge ab. Da mit zunehmender Inspirationstiefe die Compliance von Lunge und Thorax fallen, führen abfallende Flussprofile zu einem geringeren Spitzendruck bei der Beatmung. Eine exemplarische Fluss- und Druckkurve bei volumenkontrollierter Beatmung zeigt $\bullet$ Abb. 8 .

Bei volumenkontrollierter Beatmung hängt der Beatmungsdruck von der Compliance und Resistance von Thorax und Lunge ab.

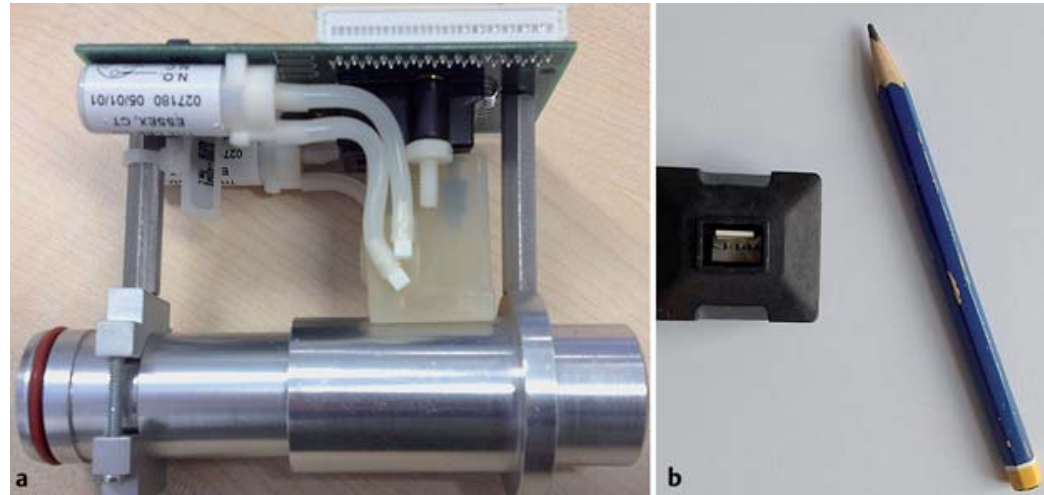

Abb. 7 Zur Flussmessung kommen zwei Sorten von Sensoren zum Einsatz: zum einen Sensoren, die das klassische Prinzip eines Pneumotachografen verwenden, bei dem über eine Druckmessung vor und nach einer Stenose der Fluss bestimmt wird (a Flusssensor aus dem Beatmungsgerät Avea, CareFusion). Alternativ kann der Fluss auch elektromagnetisch gemessen werden. Einen solchen, wesentlich kleineren Flusssensor, wie er in der Engström Carestation (GE) verwendet wird, zeigt Bild b.

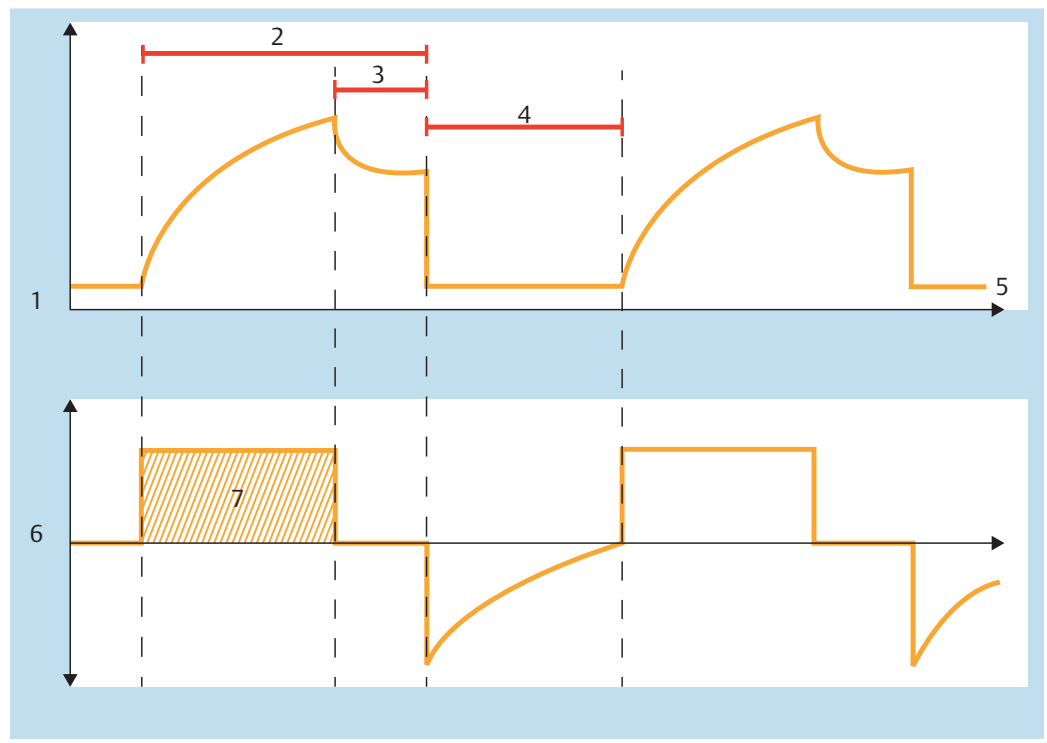

Abb. 8 Volumenkontrollierte Beatmung: $1=$ Atemwegsdruck, $2=$ Inspirationszeit des Patienten, $3=$ Inspiration des Patienten ohne Druckunterstützung durch den Ventilator, $4=$ Exspirationsphase, 5 = PEEP während der Exspiration, 6 = Flusskurve; 7 = die schraffierte Fläche (Integral von Fluss und Zeit) ergibt das aktiv vom Ventilator verabreichte Volumen. 


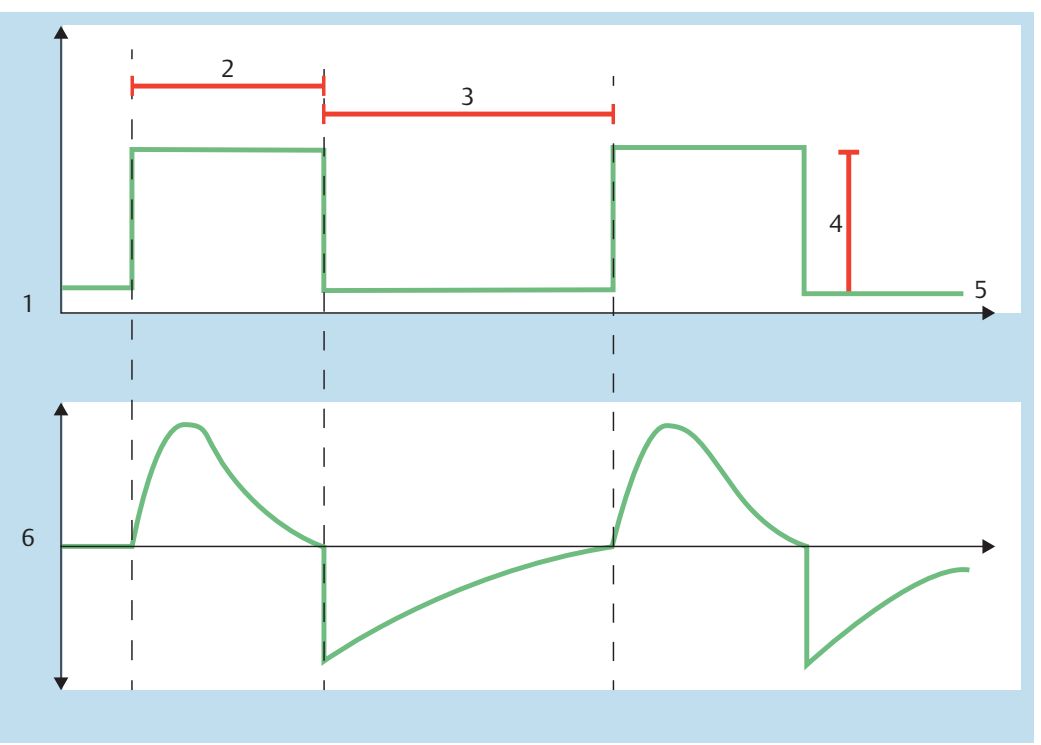

Abb. 9 Druckkontrollierte Beatmung: $1=$ Atemwegsdruck, $2=$ Inspirationszeit, $3=$ Exspirationszeit auf PEEP-Niveau, 4 = Inspirationsdruck über PEEP-Niveau, 5= PEEP , 6 = Flusskurve.

Im druckkontrollierten Modus (PCV = Pressure Controlled Ventilation) wird bei jedem vom Gerät abgegebenen Atemzug ein vorgegebener Inspirationsdruck appliziert. Wie schnell sich dieser Druck zum Beginn des Atemzuges entwickelt, wird über die Anstiegszeit, auch Flankensteilheit genannt, vorgewählt. Der Inspirationsdruck wird dann über die vorgewählte Inspirationszeit gehalten. Wie viel Volumen in dieser Zeit zum Patienten strömt, hängt wiederum von der Compliance und Resistance von Thorax und Lunge ab. Nach der Inspirationszeit schaltet das Gerät auf den PEEP-Wert. Abb.9 zeigt exemplarisch eine Fluss und Druckkurve bei druckkontrollierter Beatmung.

Bei druckkontrollierter Beatmung hängt das Tidalvolumen von der Compliance und Resistance von Thorax und Lunge ab.

\section{Regelverhalten während der Exspirationsphase \\ $\nabla$}

Bei rein kontrollierter Beatmung muss der Ventilator unabhängig von der Atemaktivität des Patienten den eingestellten PEEP-Druck während der Exspiration konstant halten.

Bei assistierter Beatmung muss der Ventilator über den Inspirationstrigger eine Atemanstrengung des Patienten adäquat erkennen und in die Inspirationsphase umschalten.

Dafür ist es erforderlich, dass auch während der Exspirationsphase Luft vom Ventilator zum Patienten (über den Inspirationsschenkel zum Y-Stück des Beatmungsschlauches) geleitet wird und von dort auch Luft über den Exspirationsschenkel zurück zum Beatmungsgerät gelangt. Diesen Luftstrom nennt man Bias-Flow. - Abb.10 zeigt exemplarisch den Aufbau. Beatmungsgeräte arbeiten entweder mit einem Drucktrigger, der bei einem Druckabfall im Beatmungssystem (hervorgerufen durch die Inspiratonsbemühung des Patienten) auslöst, oder einem Flusstrigger, der die Flussänderung misst, bzw. mit einer Kombination beider Methoden. Der Inspirationsfluss zum Patienten wird kalkuliert, indem der Ventilator die Differenz zwischen dem inspiratorischen und dem exspiratorischen Flusssensor ( $\bullet$ Abb.10) bestimmt. Die Differenz der Signale aus beiden Flusssensoren ist proportional dem Luftvolumen, das vom Patienten eingeatmet wird. Liegt dieser Fluss oberhalb der eingestellten Triggerschwelle, so löst der Trigger aus. Die Luft, die der Patient vor Auslösen des Triggers einatmet, entstammt aus dem Bias-Flow; folgerichtig muss der Bias-Flow größer sein als die eingestellte Triggerschwelle, damit diese überhaupt erreicht werden kann. Kann am Beatmungsgerät der Bias-Flow nicht entsprechend angepasst werden, so kann dies zu einer höheren Atemarbeit für den Patienten führen und unter Umständen zu einem frustranen Versuch, das Gerät anzutriggern.

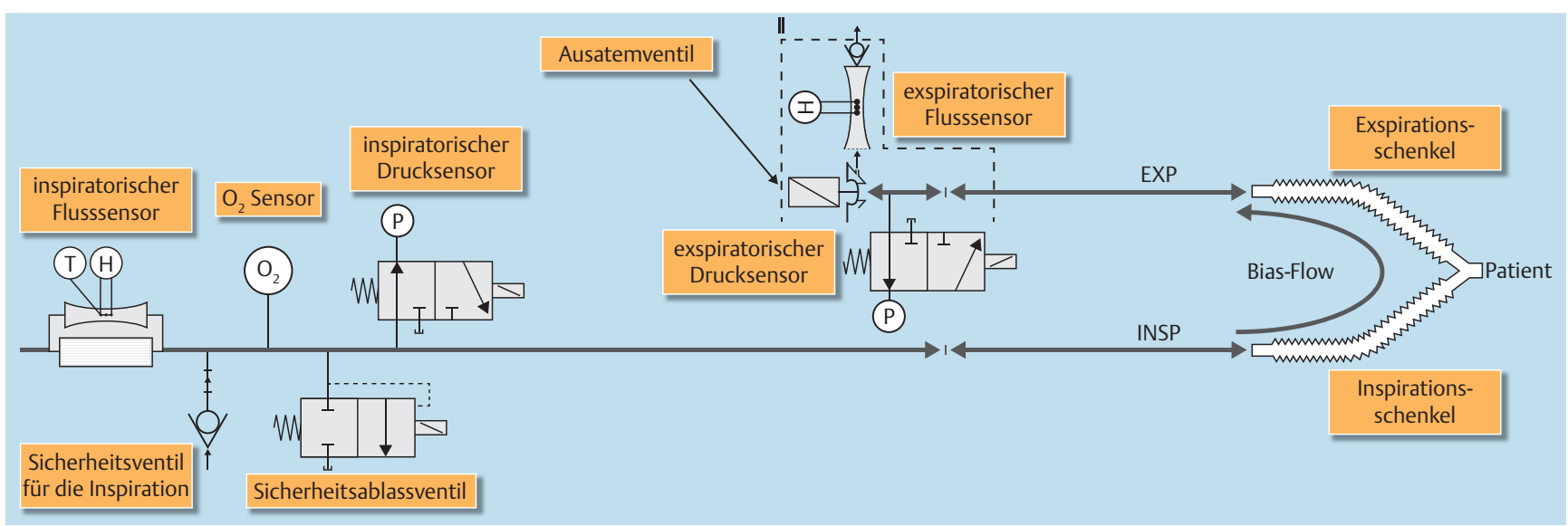

Abb.10 zeigt den In- und Exspirationsschenkel der Beatmung sowie deren Kontrollsensorik und Regelventile. Ein kontinuierlicher Fluss während der Exspiration (Bias-Flow) ist zur Aufrechterhaltung des PEEP und zur Steuerung des Inspiratonstriggers erforderlich. 
Der Bias-Flow sollte regulierbar sein; der einstellbare Range variiert zwischen den Beatmungsgeräten (z. B. 0,4-5 Liter/Min [19] bzw. 2-20 Liter/ Min [17]).

Arbeitet das Beatmungsgerät mit einem Drucktrigger, so misst der Ventilator den Druckabfall im Inspirationsschenkel (inspiratorischer Drucksensor, $\bullet$ Abb. 10). In diesem Fall wirkt ein höherer Bias-Flow dem Druckabfall entgegen und erschwert ggf. das Auslösen eines Drucktriggers.

Der eingestellte Bias-Flow sollte oberhalb der eingestellten Triggerschwelle des Flusstriggers liegen.

Zur Regelung des exspiratorischen PEEP-Wertes wird der Exspirationsschenkel mit einem entsprechenden Ausatemventil geregelt ( $\bullet$ Abb.10 und $\odot$ Abb.11). Ein im Exspirationsschenkel befindlicher Drucksensor (exspiratorischer Drucksensor, Abb. 10) misst den Druck. Mit dieser Information wird das Ausatemventil so geregelt, dass der PEEP-Wert konstant gehalten wird. Auch während der Patient aktiv exspiriert, strömt ein kontinuierlicher Bias-Flow durch den Beatmungsschlauch.

Der PEEP wird durch das Ausatemventil und einen Drucksensor im Exspirationsschenkel geregelt.

\section{Sicherheitsaspekte}

\section{$\nabla$}

\section{Überdruck im Beatmungssystem}

Als erste Sicherheitsstufe dienen hier die vom Bediener einzustellenden Alarmgrenzen. Werden diese überschritten, so ertönt ein akustisches und visuelles Alarmsignal am Respirator.

Die zweite Sicherheitsstufe besteht im softwaregesteuerten Öffnen eines Sicherheitsventils im Inspirationsschenkel sowie des Ausatemventils, wenn weitere Überdruckkriterien erfüllt sind ( $\bullet$ Abb.10). Diese Kriterien können ein längerfristiges (mindestens 5 Sekunden) Überschreiten der Druckalarmobergrenze [19] oder das Erreichen eines separat eingestellten kritischen Druckwertes sein [17].

Die dritte Sicherheitsstufe besteht aus einem rein mechanischen Überdruckventil, welches automatisch öffnet, wenn ein ventilspezifischer Druck erreicht wird. Dieser kritische Druck lag bei den untersuchten Geräten bei 120 [17] bzw. 125 [19] mbar.

Überdruckalarme schützen nicht vor der Applikation von zu hohen Beatmungsdrücken. Die softwaregesteuerte Öffnung der Ventile bei Überdruck bzw. die Öffnung mechanischer Überdruckventile wird herstellerspezifisch geregelt.

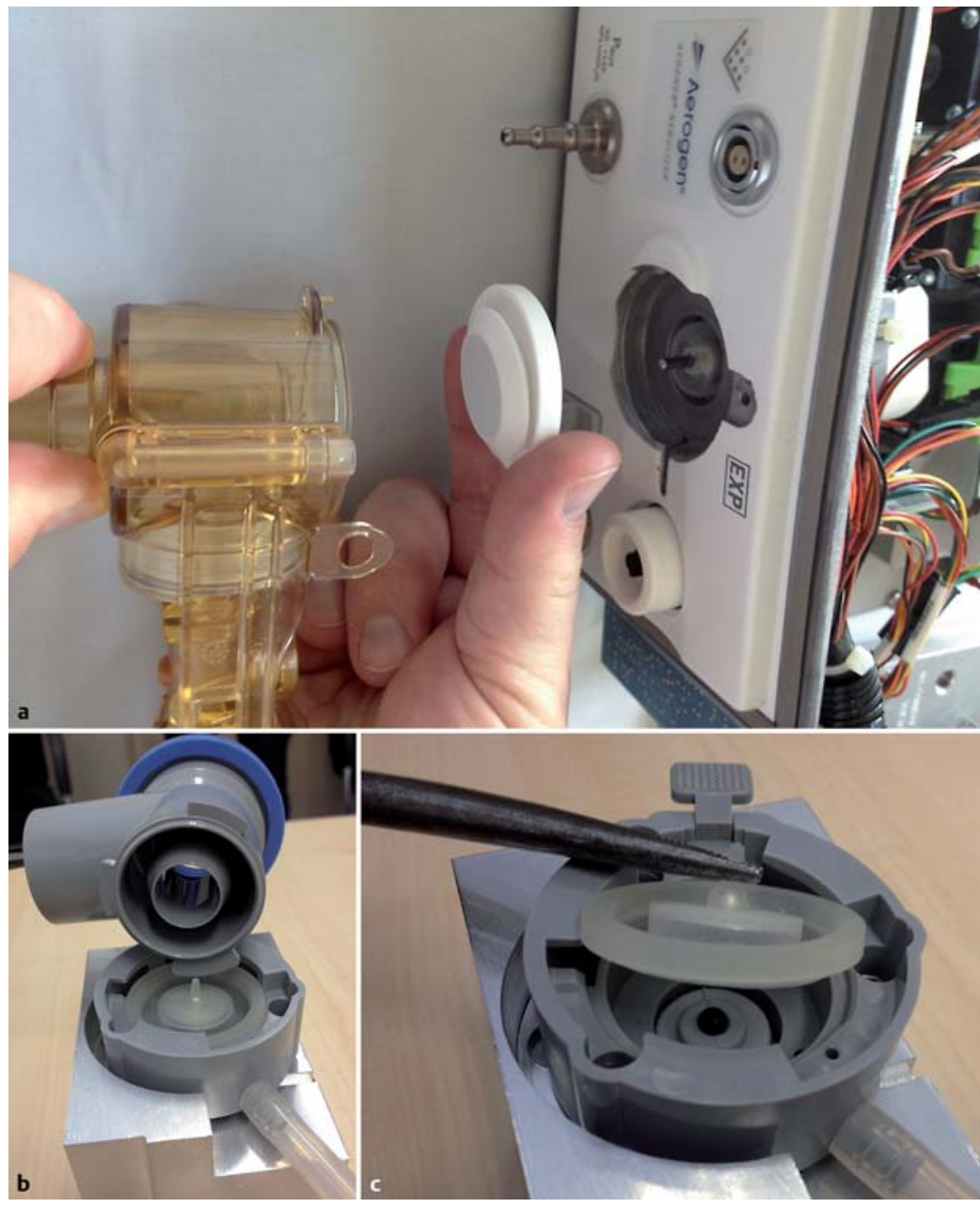

Abb.11 zeigt Ausatemventile von Beatmungsgeräten. Von der Bauart her handelt es sich dabei um Ventile, die über eine Membran geregelt werden. Bild a zeigt das Ventil des Beatmungsgerätes Engström, Carestation (GE) Bilder b und c das entsprechende Ventil aus der Avea, CareFusion.

\section{Unterdruck im Beatmungssystem}

Als erste Sicherheitsstufe dient hier ebenfalls ein akustisches und visuelles Signal am Ventilator, falls der eingestellte Grenzwert unterschritten wird.

Zusätzlich haben alle Beatmungsgeräte einfache mechanische, unidirektionale Membranventile, über die der Patient Luft einatmen kann, falls der geräteinterne Druck unterhalb des Umgebungsdruckes (Raumdruck) abfällt ( $\bullet$ Abb.12). Der Strömungswiderstand eines drucklosen Beatmungsgerätes erhöht dabei natürlich die Atemarbeit des Patienten.

Bei einem Druckabfall im Gerät kann der Patient eigenständig durch das Schlauchsystem des Beatmungsgerätes atmen. Dies wird durch einfache unidirektionale Membranventile gewährleistet. Eine Sauerstoffbeimischung findet hierbei nicht mehr statt.

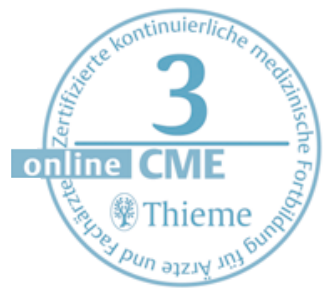



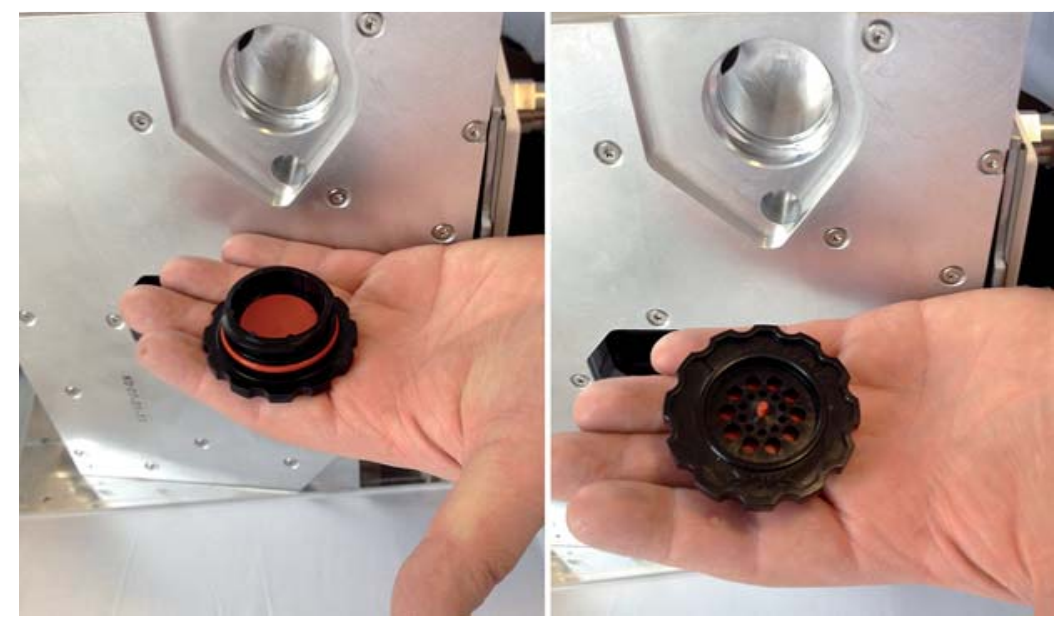

Abb.12 Die beiden Bilder zeigen das Sicherheitsventil für die Inspiration aus der Engström Carestation (GE). Dieses Ventil ermöglicht ein freies Atmen des Patienten, falls es zu einem Druckabfall im Beatmungsgerät kommt.

\section{Danksagung}

Die Autoren danken Herrn Marc Missbach und Herrn Peter Claassen von der Firma GE sowie Herrn Kay Krüger und Herrn Charles Hall von der Firma CareFusion für ihre Unterstützung bei der Erstellung dieses Artikels.

\section{Interessenkonflikt \\ $\nabla$}

D. Heyse und S. Böckling: Firma ResMed.

K. Siemon und D. Dellweg: Firma ResMed und Firma Weinmann.

G. Schürholz gibt an, dass kein Interessenkonflikt besteht.

\section{Literatur}

1 Biermann A, Geissler A. Beatmungsfälle und Beatmungsdauer in deutschen Krankenhäusern: Eine Analyse von DRG-Anreizen und Entwicklungen in der Beatmungsmedizin. Berlin: Technische Uni Berlin; 2014; https:/ www.mig.tu-berlin.de/fileadmin/a38331600/sonstiges/ WPS_vol_7_final.pdf

2 Hari MS, Jennings $M$. Flow sensor fault causing ventilator malfunction. Anaesthesia 2005; 60: 1049-1050

3 Krishna Kumar B, Ravi M, Dinesh $K$ et al. Ventilator malfunction. J Anaesthesiol Clin Pharmacol 2011; 27: 576

4 Gravenstein D, Wilkhu H, Liem EB et al. Aestiva ventilation mode selector switch failures. Anesth Analg 2007; 104: $860-862$

5 Bourke DL, Tolentino D. Inadvertent positive end-expiratory pressure caused by a malfunctioning ventilator relief valve. Anesthesia and analgesia 2003; 97: 492-493

6 Cassidy Cl, Smith A, Arnot-Smith I. Critical incident reports concerning anaesthetic equipment: analysis of the UK National Reporting and Learning System (NRLS) data from 2006-2008*. Anaesthesia 2011; 66: 879-888

7 Chatwin M, Heather S, Hanak A et al. Analysis of home support and ventilator malfunction in 1,211 ventilatordependent patients. Eur Respir ] 2010; 35: 310 - 316

8 Daiy YP, Shu CC, Lui PW et al. Pulmonary barotrauma caused by expiratory check valve malfunction of anesthetic machine. Ma zui xue za zhi = Anaesthesiologica Sinica 1992; 30: $51-54$

9 di Paolo M, Evangelisti L, Ambrosino N. Unexpected death of a ventilator-dependent amyotrophic lateral sclerosis patient. Revista portuguesa de pneumologia 2013; 19: $175-178$

10 Duckett JE, Hice C, Smith TC. Hazard of a ruptured exhalation mushroom diaphragm. Crit Care Med 1980; 8: $750-751$

11 Eisenkraft JB. Potential for barotrauma or hypoventilation with the Drager AV-E ventilator. Journal of clinical anesthesia 1989; 1: 452-456

12 Gaines GY 3rd, Rees DI. Ventilator malfunction - another cause. Anesthesiology 1984; 60: 260-261

13 Lehmann DW, Engelbrecht K, Radke ]. [Functional failure of an inspiratory Draeger-circuit system. An example of the fundamental problem of borderline damage to a component part in anesthesia and intensive care]. Der Anaesthesist 1997; 46: 801-804

14 Mahajan R, Gulati S, Jatinder M. Ventilator malfunction due to Luer lock misconnection. Anaesthesia 2014; 69: $791-792$

15 Organizations, J.C.o.A.o.H.C. Preventing ventilator-related deaths and injuries. Sentinel Event Alert 2002; 25

16 Arzneibuchkommission. Europäisches Arzneibuch. Aer medicalis. 7. Vol. Deutscher Apotheker Verlag; 2013

17 Inc., D.-O. Engström Carestation Technical Reference Manual. Madison; 2011

18 Smith F. Schematic Diagram Pneumatic, Avea, CareFusion. San Diego; 1999

19 CareFusion Avea. Ventilator System Operator's Manual. 2011

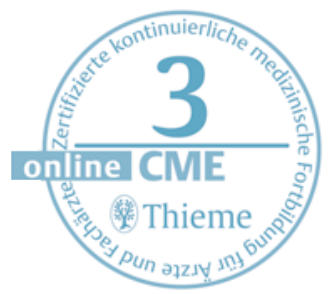




\section{CME-Fragen Technische Aspekte der Beatmungsmedizin}

Welche Aussage zur Bedieneinheit ist richtig?

A Hier sollten alle relevanten Parameter intuitiv und übersichtlich einzustellen sein. Die Tasten und Knöpfe sollten so konstruiert sein, dass ein versehentliches Verstellen der Parameter nicht möglich ist.

B Ein monochromes Display ist besser als ein farbig gestaltetes.

C Hier findet die Gaseinleitung in das Gerät statt.

D Um Strom zu sparen, stellt sich das Display im laufenden Betrieb in den Schlafmodus.

E Nach Ein- oder Umstellung eines Parameters ist keine Bestätigungstaste erforderlich.

Welche Aussage ist richtig? Das pneumatische System ...

A ist eine baugleiche Komponente bei allen Geräteherstellern.

B regelt Druck, Fluss und Sauerstoffkonzentration des Atemgases.

C kann durch den sogenannten Blender Druckschwankungen des Atemgases ausgleichen.

D enthält mit dem Akkumulator ein Sauerstoffreservoir.

E wird ausschließlich mit externer Druckluft betrieben.

A Der Arbeitsdruck im Beatmungsgerät entspricht dem Druck des zuführenden Anschlusses für medizinische Gase (in der Regel 5 bar bei Wandanschlüssen).

B Der Arbeitsdruck im Beatmungsgerät muss sehr hoch ( $>5$ bar) sein, damit bei plötzlichen Druckabfällen der Atemgasfluss zum Patienten ausreichend hoch gehalten werden kann.

c Ein zusätzlicher patientennaher Druckspeicher im Beatmungsgerät (Akkumulator) kann bei einem plötzlichen Druckabfall einen ausreichenden Atemgasfluss zum Patienten aufrecht erhalten.

D Ein zusätzlicher patientennaher Druckspeicher im Beatmungsgerät (Akkumulator) braucht nur mit Raumluft gefüllt zu werden, da er nur im Notfall Verwendung findet.

E Bei Geräten mit internem Kompressor können auch ohne zusätzlichen Sauerstoffanschluss beliebig hohe Sauerstoffkonzentrationen erzeugt werden.
4 Welche Aussage ist falsch? Für die Leitung von medizinischen Gasen im Beatmungsgerät gelten folgende Aussagen:

A Der Strömungswiderstand des luftleitenden Systems im Beatmungsgerät sollte so hoch wie möglich sein, damit keine starken Druckschwankungen im System auftreten können.

B Der Strömungswiderstand des luftleitenden Systems im Beatmungsgerät sollte möglichst gering gehalten werden, damit der im Gerät verwendete Druck zu einem ausreichenden Fluss des Atemgases zum Patienten führt.

c Für die Leitung des Atemgases im Gerät zwischen den einzelnen Komponenten werden häufig Schlauchverbindungen verwendet.

D Es gibt Beatmungsgeräte, die zur internen Atemgasleitung keine Schläuche verwenden, sondern in eine Aluminiumbodenplatte gefräste Kanäle, welche wiederum sehr stabil und wartungsfrei sind.

E Ein zusätzlicher patientennaher Druckspeicher im Beatmungsgerät (Akkumulator) sollte immer mit einem Atemgasgemisch durchflutet werden, welches die eingestellte Sauerstoffkonzentration enthält, damit bei Druckschwankungen mit dem korrekten Atemgas beatmet wird.

5 Welche Aussage ist falsch? Für die Steuerung des Atemgases zum Patienten gilt:

A Die Steuerungseinheit zur Regelung des Atemgasflusses zum Patienten besteht aus Flussventil, Flusssensor und Drucksensor.

B Bei einem internen Druckspeicher muss dieser hinter den Regelventilen zur Atemgasmischung liegen, damit dieser immer mit dem richtigen Atemgasgemisch durchflutet werden kann.

C Die Steuerungseinheit für Druck und Fluss des Atemgases zum Patienten muss, in Flussrichtung gesehen, dann hinter dem Anschluss für den internen Druckspeicher liegen.

D Eine Kommunikation und zentrale Rechen- und Regeleinheit der einzelnen Flussventile, Flusssensoren und Drucksensoren ist nicht erforderlich, da die gewünschte Atemgasmischung und die notwendigen Druckprofile durch die Anordnung der Schlauchsysteme bzw. Kanäle im Beatmungsgerät ausreichend eingestellt sind.

E Die gewünschte inspiratorische Sauerstoffkonzentration im Beatmungsgerät wird durch einen Blender erreicht, welcher die Zuführung der medizinischen Atemgase regelt und somit korrekt vermischt. 
6 Welche Aussage ist richtig? Es gibt verschiedene Beatmungsmodi:

A Bei einem druckkontrollierten Modus ( $\mathrm{PCV}=$ pressure controlled ventilation) hängt das Atemzugvolumen bzw. Tidalvolumen nur von den eingestellten Drücken ab und ist daher bei jedem (Be-) Atmungszug konstant.

B Bei einer Volumen-kontrollierten Beatmung (VCV $=$ volume controlled ventilation) ist das Flussprofil sinnvollerweise immer konstant während eines (Be-)Atmungzuges.

C Abfallende Flussprofile können bei VCV helfen, den Beatmungsspitzendruck zu reduzieren, da bei zunehmender Inspirationstiefe die Compliance von Lunge und Thorax fällt.

D Bei der PCV ist der vorgegebene Inspirationsdruck von Anfang bis zum Ende des (Be-)Atmungszuges gleich hoch.

E Durch die Einstellung einer Flankensteilheit bzw. Druckanstiegszeit kann die Belastung der Lunge in der PCV, insbesondere bei hohen Beatmungsdrücken oder bei steifen Lungen, reduziert werden. Der eingestellte Inspirationsdruck wird hierbei aber nicht mehr beachtet.

Für die Beatmungsmodi gilt:

A Die VCV ist die beste Beatmungsform, da ja immer das eingestellte Volumen pro Beatmungszug oder pro Minute erreicht wird.

B Die PCV ist die beste Beatmungsform, da es zu keinen gefährlichen Druckspitzen kommen kann und trotzdem eine konstante Beatmung und Ventilation garantiert wird.

C Bei der VCV muss ein enges Alarmregime beachtet werden, weil sonst zum Erreichen der eingestellten Volumina pathologisch hohe Beatmungsdrücke entstehen können oder bei Verhinderung von pathologischen Beatmungsdruckspitzen die eingestellten bzw. notwendigen Beatmungsvolumina nicht erreicht werden.

D Bei der PCV spielen Compliance und Resistance von Lunge und Thorax für das zuführbare Volumen pro Zeit keine Rolle.

E Bei der VCV spielen Compliance und Resistance von Lunge und Thorax für die maximalen Druckspitzen keine Rolle.
8 Welche Aussage zum Regelverhalten während der Exspirationsphase trifft zu?

A Bei rein kontrollierter Beatmung muss der Exspirationstrigger besonders sensibel eingestellt werden.

B Bei assistierter Beatmung ist der Inspirationstrigger vernachlässigbar.

c Der Bias-Flow ist eine einstellbare Größe, welche oberhalb der eingestellten Triggerschwelle des Flusstriggers liegen sollte.

D Der PEEP wird im Druckspeicher generiert.

E Bei aktiver Exspiration bricht der Bias-Flow im Beatmungsschlauch ab.

Was passiert bei einem Überdruck im Beatmungssystem?

A Das Beatmungsgerät schaltet sich ab.

B Neben akustischen und optischen Alarmierungen wird ab einer bestimmten Druckgrenze ein Sicherheitsventil im Inspirationsschenkel und das Ausatemventil geöffnet.

C Ein mechanisches Überdruckventil ist nicht erforderlich.

D Das mechanische Überdruckventil der Beatmungsgeräte schützt den Patienten vor Druckschäden der Lunge.

E Die Öffnung der mechanischen Überdruckventile bei Überdruck im System ist einheitlich geregelt.

Welche Aussage zum Unterdruck (kritischer Druckabfall) im Beatmungssystem ist richtig?

A Bei Druckabfall im Gerät ermöglichen unidirektionale Membranventile dem Patienten die Atmung durch das Schlauchsystem.

B Im Falle eines Druckabfalls ermöglichen Bidirektionale Membranventile dem Patienten die Atmung durch das Schlauchsystem.

C Der Strömungswiderstand des drucklosen Beatmungsgerätes beeinflusst die Atemarbeit des Patienten nicht.

D Im Falle eines Unterdrucks im Beatmungssystem übernimmt der Akkumulator automatisch die Beatmung.

E Der Unterdruckalarm ist leiser als der Überdruckalarm. 